\title{
INVERSE SYSTEMS OF ABSTRACT LEBESGUE SPACES
}

\author{
BRUCE CHRISTIANSON
}

(Communicated by William J. Davis)

\begin{abstract}
We show that inverse limits exist in the category of $L^{1}$ spaces and positive linear contractions between them. This result generalizes the wellknown classical results for inverse systems of Choquet simplexes and of $L$-balls, but our proof is simple and more purely geometrical. The result finds physical application in the study of random fields.
\end{abstract}

Let $Z$ be a Banach lattice with positive cone $C$ and norm $\|\cdot\|$. We say that $Z$ is an $A L$-space (short for Abstract Lebesgue space) iff $\|\cdot\|$ is affine on $C$. See [7, $\S 8$, p. 112] for further information about $A L$-spaces.

Let $f$ be a linear map between two $A L$-spaces $Z_{2}$ and $Z_{1}$. We say that $\mathrm{f}$ is a contraction iff $\|f x\|_{1} \leq\|x\|_{2}$ for all $x \in C_{2}$. A positive linear contraction $f: Z_{2} \rightarrow Z_{1}$ is called an $A L$-morphism .

Note that for an $A L$-morphism $f$ we have $\|f x\|_{1} \leq\|x\|_{2}$ for all $x \in Z_{2}$ since

$$
\|f x\|_{1}=\left\||f x|_{1}\right\|_{1} \leq\left\|f|x|_{2}\right\|_{1} \leq\left\||x|_{2}\right\|_{2}=\|x\|_{2} ;
$$

however, an $A L$-morphism need not be a lattice homomorphism, an isometry, or even one-to-one.

Let $D$ be a directed set, and suppose that we have an $A L$-space $Z_{i}$ associated with each $i \in D$ and an $A L$-morphism $f_{j i}: Z_{i} \rightarrow Z_{j}$ associated with each pair $i, j \in D$ where $i>j$.

If this system is consistent in the sense that

$$
f_{j i}=f_{j k} f_{k i} \quad \text { for all } i>k>j \text { in } D,
$$

then we call $\left(Z_{i}, f_{j i}\right)_{D}$ an inverse $A L$-system.

Now suppose further that $Z$ is an $A L$-space, and $f_{i}: Z \rightarrow Z_{i}$ for $i \in D$ are $A L$-morphisms satisfying

$$
f_{j}=f_{j i} f_{i} \quad \text { for all } i>j \text { in } D .
$$

Then we call $\left(Z, f_{i}\right)_{D}$ a prefix of $\left(Z_{i}, f_{j i}\right)_{D}$.

Received by the editors December 9, 1989 and, in revised form, January 9, 1990.

1980 Mathematics Subject Classification (1985 Revision). Primary 46B30, 46M40. 
If every other prefix $\left(Y, g_{i}\right)_{D}$ of $\left(Z_{i}, f_{j i}\right)_{D}$ factors through $\left(Z, f_{i}\right)_{D}$ in the sense that there exists an $A L$-morphism $g: Y \rightarrow Z$ with

$$
g_{i}=f_{i} g \quad \text { for all } i \in D \text {, }
$$

then we call $\left(Z, f_{i}\right)_{D}$ the inverse $A L$-limit of $\left(Z_{i}, f_{j i}\right)_{D}$.

Theorem. For every inverse AL-system $\left(Z_{i}, f_{j i}\right)_{D}$ the inverse $A L$-limit $\left(Z, f_{i}\right)_{D}$ exists and is unique.

Proof. Clearly the inverse $A L$-limit is unique up to $A L$-isomorphism if it exists. We demonstrate existence by constructing the inverse $A L$-limit explicitly.

Let $\Pi Z$ stand for $\prod_{i \in D} Z_{i}$ and let $\pi_{i}: \Pi Z \rightarrow Z_{i}$ denote the coordinate projections. Write $x_{i}$ for $\pi_{i} x$ where $x \in \Pi Z$.

Define

$$
\begin{gathered}
\|x\|=\sup _{i \in D}\left\|x_{i}\right\|_{i} \text { for } x \in \Pi Z, \\
Z=\left\{x \in \Pi Z:\|x\|<\infty \text { and } f_{j i} x_{i}=x_{j} \text { for all } i>j \in D\right\}, \\
C=\left\{x \in Z: x_{i} \in C_{i} \text { for all } i \in D\right\},
\end{gathered}
$$

and let $f_{i}: Z \rightarrow Z_{i}$ be the restriction of $\pi_{i}$ to $Z$.

We shall show that $Z$ equipped with $C$ and $\|$.$\| is an A L$-space. First we note that $(Z, C)$ is a vector lattice with sum and modulus given by

$$
(x+y)_{j}=x_{j}+y_{j}, \quad(|x|)_{j}=\lim _{i>j} f_{j i}\left|x_{i}\right|_{i}
$$

where $|\cdot|_{i}$ denotes modulus in $Z_{i}$ and the limit is in $\|\cdot\|_{j}$.

To see that the definition of modulus makes sense we use a property of $A L$ spaces, that every monotone increasing norm-bounded net converges in norm to its least upper bound. This is easy to prove directly, or see [7, 8.2, p. 113].

Clearly $f_{j i}\left|x_{i}\right|_{i}$ is a monotone increasing sequence in $Z_{j}$ with norm bounded by $\|x\|$. Hence this sequence is norm convergent in $Z_{j}$, and indeed $f_{j i}(|x|)_{i}=$ $(|x|)_{j}$ and $\||x|\|=\|x\|<\infty$. Thus $|x| \in Z$ and is easily seen to be the least upper bound in $Z$ of $\{x,-x\}$.

It is now easy to verify that $\|\cdot\|$ is a lattice norm for $Z$ and is additive on $C$. To show the norm complete under these conditions it suffices to prove that every monotone increasing norm bounded net $\left(x^{(n)}\right)$ in $Z$ has a least upper bound $x[7,8.2$, p. 113]. But this is easy, set

$$
x_{j}=\lim _{n} x_{j}^{(n)} \quad \text { for } j \in D
$$

and argue as in the previous paragraph to show that $x \in Z$ and is the required least upper bound.

This establishes that $Z$ is an $A L$-space. The $f_{i}$ are obviously $A L$-morphisms so $\left(Z, f_{i}\right)_{D}$ is a prefix of $\left(Z_{i}, f_{j i}\right)_{D}$. For any other prefix $\left(Y, g_{i}\right)_{D}$ define $g: Y \rightarrow Z$ by $(g y)_{i}=g_{i} y$. Verification of the factorization property is now routine. Hence $\left(Z, f_{i}\right)_{D}$ is the inverse $A L$-limit of $\left(Z_{i}, f_{j i}\right)_{D}$. 
If the dual of a Banach space can be ordered in such a way as to make it an $A L$-space, then the unit ball of the dual endowed with the weak-* topology is called an $L$-ball [5].

If all the $A L$-spaces $Z_{i}$ are dual spaces of this form then our theorem implies the result of D. A. Edwards [3, Corollary 5, p. 231] that the inverse limit of a system of $L$-balls is an $L$-ball.

If in addition each $S_{i}=\left\{x \in C_{i}:\|x\|_{i}=1\right\}$ is a weak-* closed face of the corresponding $L$-ball, then the $S_{i}$ are Choquet simplexes.

Conversely (see for example [2, Corollary 3, p. 411]) every Choquet simplex is affine homeomorphic to a simplex of this form. Each affine continuous map $f_{j i}$ from $S_{i}$ into $S_{j}$ then extends naturally to an $A L$-morphism from $Z_{i}$ into $Z_{j}$ (although this $A L$-morphism may fail to be an isometry.)

Thus our theorem also implies the result of F. Jellett, E. B. Davies, and G. F. Vincent-Smith $[4,1]$ that the inverse limit of a system of Choquet simplexes is a Choquet simplex.

Results of this type are of interest in statistical mechanics (see for example $[8, \S 4.3])$. There, the spaces of measures used to underlie the representations of Gibbs states for Föllmer specifications are $A L$-spaces, and the corresponding substochastic kernals are $A L$-morphisms.

Often we are concerned only with geometric properties which are intrinsic to the relevent inverse limit space (see for example $[6, \S 1.6]$ ) and in such cases our theorem establishes sufficient structure to determine the phases.

\section{REFERENCES}

1. E. B. Davies and G. F. Vincent-Smith, Tensor products, infinite products and projective limits of Choquet simplexes, Math. Scand. 22 (1968), 145-164.

2. D. A. Edwards, On the homeomorphic affine embedding of a locally compact cone into a Banach dual space endowed with the vague topology, Proc. London Math. Soc. (3) 14 (1964), 399-414.

3. __ Systèmes projectifs d'ensembles convexes compact, Bull. Soc. Math. France 103 (1975), 225-240.

4. F. Jellett, Homeomorphisms and inverse limits of Choquet simplexes, Math. Z. 103 (1968), 219-226.

5. A. J. Lazar, The unit ball in conjugate $L^{1}$ spaces, Duke Math. J. 39 (1972), 1-8.

6. L. Le Cam, Asymptotic methods in statistical decision theory, Springer Series in Statistics, Springer-Verlag, Berlin, 1986.

7. H. H. Schaefer, Banach lattices and positive operators, Grundlehren. Math. Wissen., vol. 215, Springer-Verlag, Berlin, 1974.

8. G. Winkler, Choquet order and simplices with applications in probabilistic models, Lecture Notes in Math., vol. 1145, Springer-Verlag, Berlin, 1985. 